\title{
Safety and Efficacy of Saccharomyces boulardii for the management of Diarrhoea in Indian children
}

\author{
Dr. Mayuresh Kiran ${ }^{1 *}$, Mr. Lalit Pawaskar ${ }^{2}$ \\ ${ }^{1}$ General Manager, Medical Services, Centaur Pharmaceuticals Pvt. Ltd. \\ ${ }^{2}$ Research Associate, Pharmacovigilance, Centaur Pharmaceuticals Pvt. Ltd.
}

\begin{abstract}
Objective: S. boulardii seem to be an assuring biotherapeutic agent for the amelioration of the course of diarrhoea in children when used therapeutically. S. boulardii mediates responses resembling the protective effects of the normal healthy gut flora. Our objective was to access the safety and efficacy of S. boulardii on childhood diarrhea.

Methodology: Total 210 patients were enrolled out of which 184 patients completed the study S. boulardii sachets ( 5 million CFU 282.5mg) were given to the patients enrolled in the study. Efficacy and safety assessment was made by quantitating the Frequency, Intensity and Consistency of stools.

Results: Stool frequency decreased from 9.005 to 2.7 at the end of 5 days. Severity scale was extrapolated which interestingly showed zero patients with severe diarrhoea. There was a reduction in the duration of diarrhoea along with stool consistency where in majority patients with watery stool came down to 2 from 136.

Conclusions: Saccharomyces boulardii is safe and efficacious in the treatment of diarrhoea in Indian children.
\end{abstract}

Keywords: Saccharomyces boulardii, Diarrhoea

\section{Introduction:}

Worldwide, diarrhoeal diseases are a major health problem and leading cause of death in children under 5 years old. According to World Health Organisation (WHO) Diarrhoea is the passage of 3 or more loose or liquid stools per day, or more frequently than is normal for the individual ${ }^{1}$. Diarrhea is not a disease but a symptom therefore definitions of diarrheal episodes are not based on etiology but duration of symptoms ${ }^{2}$. Acute watery diarrhoea, acute bloody diarrhoea, persistent diarrhoea etc are clinical types of diarrhoea.

Globally, there are nearly 1.7 billion cases of childhood diarrhoeal disease and 750,000 deaths in children under the age of 5 per year. Diarrhoea is a dominant cause of malnutrition, and malnourished children are more likely to fall ill from the same. Although India has made steady progress in reducing deaths in children younger than 5 years the proportional death accounted by diarrheal disease still remains high killing an estimated 300,000 children each year accounting for $13 \%$ deaths in this age-group ${ }^{3}$.

Last couple of decades has seen preeminent understanding of pathogenesis and simple means of management of diarrhoea. Assorted modalities of interventions have been used in different parts of the world to amend the diarrhoeal morbidity and mortality which include newly formulated oral rehydration salt (ORS), zinc, anti-diarrhoeal, antibiotics, antisecretory, antispasmodics and anti-emetics ${ }^{4}$. Some of these modalities later confirmed to have variable harmful effects including increased duration of diarrhoea or worsening of the same, adverse effects on gut motility leading to other untoward effects ${ }^{3}$.

Gastrointestinal disease is often a repercussion of a myriad of factors, which agitate the bowel's complex ecosystem. The concept of regulating bacterial activities, directed towards repairing gut microbial function, has a long history ${ }^{5}$. The use of yoghurt (as probiotic) in the treatment of diarrhoea has been popular for a long time. It is now perceived that the most commonly used method of persuading the gut flora composition is that of probiotics in the pediatric population ${ }^{6}$. A probiotic is a living microorganism purported to have a health benefit on the host by treating or preventing infections owing to strains of pathogens. They are nothing but bio-therapeutic agents now registered as drugs ${ }^{7}$. 
Numerous probiotic agents have been studied for the management of diarrhoeal disease. In particular, the management and prevention of acute viral diarrhoea, the treatment of Clostridium difficile diarrhoea, likewise the control of antibiotic-associated diarrhoea seem to be areas of momentous potential benefit ${ }^{8}$. A few agents, including Lactobacillus GG, Lactobacillus acidophilus, Lactobacillus casei, Bifidobacterium ssp, Streptococcus ssp, Bacillus Clausii and Saccharomyces boulardii (S. boulardii), seem to be assuring agents for the amelioration of the course of diarrhoea in children when used therapeutically. Amidst these, all are bacteria except $\mathrm{S}$. boulardii, which is yeast ${ }^{8}$.

Experimental and preclinical studies of S. boulardii have indicated an anti-inflammatory, enzymatic, metabolic, antimicrobial and antitoxinic activity. S. boulardii secretes a 54-KDa protease which neutralises certain bacterial toxins; S. boulardii stimulates an immune response in the intestinal mucosa. It has a trophic effect by augmenting the metabolic function of the mucosa. It releases polyamines, which are involved in stimulating the enzymatic activity of the colonic mucosa ${ }^{9}$.

Prof. Gregor Reid Probiota 2014 stated that multi-strain products are less effective than products with single strain since the strains inhibit each other and are very likely to compete for survival, food, attachment site on the whole for the right to live in the gut, which defeats the purpose of taking a probiotic in the first place ${ }^{10}$.

A Cochrane review on pediatric antibiotic-associated diarrhoea (ADD) implied a protective association of probiotic use in hindering antibiotic-associated diarrhoea in children. It was seen that among the various probiotics evaluated, Saccharomyces boulardii at 5 to 40 billion colony forming units/day appeared suitable for preventing $\mathrm{AAD}$ in children receiving antibiotics ${ }^{11}$. A study from BMJ has conducted randomized clinical trial of five different probiotics preparation \& concluded that all probiotics are not same regarding efficacy and depend on the strain of probiotic used ${ }^{12}$.

There are different probiotic preparations that are available in Indian market, and S.boulardii is one of the majorly prescribed probiotic. This study was undertaken to assess the safety and efficacy of S.boulardii on Indian children in the treatment of diarrhoea.

\section{Materials and methods}

This Phase IV clinical trial enrolled 12 Pediatric centres in various cities in India for the study. This study was conducted from January 2017 to April 2017. Total 210 patients were recruited for the study out of which 184 patients completed the study. 26 patients were lost to follow-up.

\section{Inclusion and exclusion criteria}

Patients with confirmed diagnosis of diarrhoea were included in the study. The study subsumed patients of both gender above 1 year. Only the patients with guardians who would strictly cohere to the protocol were recruited for the study. Patients with known hypersensitivity to S.boulardii and other probiotics and seriously ill patients were excluded from the study.

\section{Study intervention}

Saccharomyces boulardii sachets ( 5 million CFU 282.5mg) were provided by the sponsor free of cost to the patients enrolled in the study. Study dosage and administration - Patients were asked to take 2-3 sachets per day for 5 days.

\section{Study procedure}

The study stretch was decided to be 5 days to test safety and efficacy of the S.boulardii. Patients of diarrhoea satisfying the inclusion and exclusion criteria were recruited for the study. Medical history was taken and physical examination (including respiratory rate, blood pressure in the sitting position, oral temperature and pulse rate) was conducted by the investigators. Investigators holding post-graduate degree in Pediatric speciality were involved in conduction of this study. Patients were given free samples in the dose of one to two satches per day for a study period of 5 days with $8 \mathrm{hrs}$ interval. Patients were asked to maintain a symptoms diary and note any adverse events occurring during the study duration. Three visits were outlined for all the patients recruited in this study - V1 (baseline visit) on day 1 before treating patient with the study medication, V2 (revaluation visit) on day 3 and V3 (last or conclusion visit) on day 5.Frequency, Intensity and Consistency of stool were noted at each visit along with physical examination and medical history. Patients were instructed to keep a diary of daily symptoms. Investigators were asked to discontinue the study 
drug in case of severe adverse event and with discretion, clinical experience contingent upon mild or moderate adverse events.

\section{Concomitant therapy}

Patients were advised to take ORS AND Zinc with S.boulardii sachets. Use of antibiotics was recommended to be avoided as far as possible. Use of Antisecretory medications like Loperamide and Diphenoxylate + Atropine was strictly prohibited during the study period.

\section{Regulatory and Ethical matters}

S.boulardii is freely available in India and is prescribed by the medical professionals and many of the times also taken OTC. All the participated patients in the study have read and voluntarily signed the informed consent form (ICF). This study was conducted by following all the rules and guidelines as per schedule Y. The ICF, protocol, CRF, investigators $\mathrm{CV}$, investigators undertaking, ethics committee registration certificates and investigators medical registration certificates (including post-graduation certificates) were submitted to the office of DCGI (Drug Controller General of India), Central Drugs Standard Control Organization (CDSCO).

\section{Result:}

184 patients were analysed in the study, on day 3(Visit 2) after taking Saccromyces boulardii there was a significant reduction in reported number of stools or stool frequency to 5.27 from 9.005(Day 1).On day 5 (Visit 3) it was further reduced and had a significantly lower frequency of 2.7 episodes. There was a significant reduction seen with the treatment of Saccharomyces boulardii from Day 1 to Day 5.

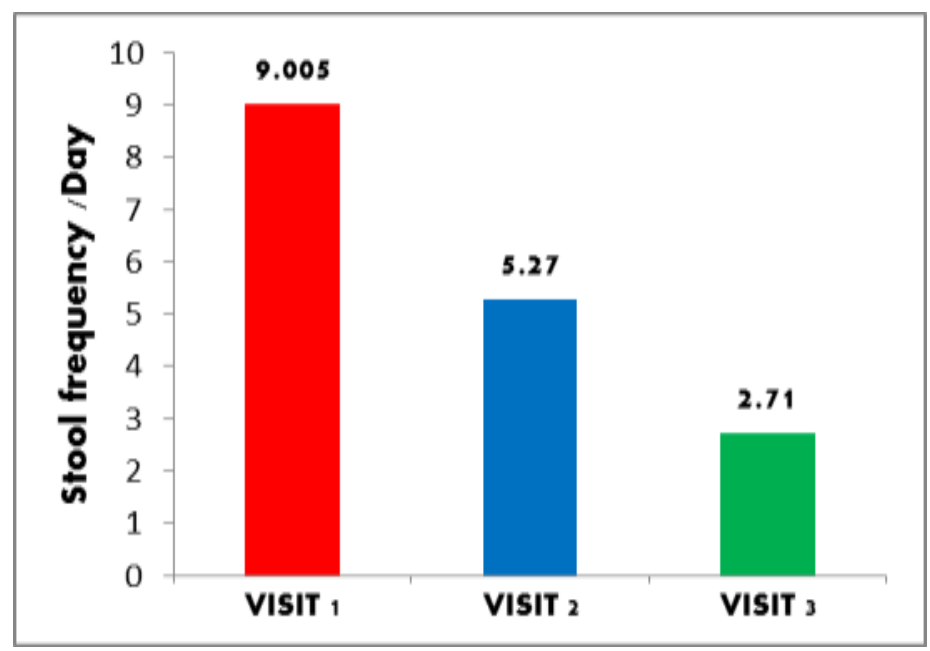

Fig.1 Stool frequency at each visit

The data with regards to the stool frequency/day was extrapolated to Diarrhoea severity scale of $0-3$ stools/days-Normal or No diarrhoea; 4-7 mild; 8-9 moderate and 10 and above severe. 61 patients had mild diarrhoea, 32 patients had moderate and 76 patients had severe at Visit 1 . The cases of severe diarrhoea were reduced to 17 patients, moderate diarrhoea 23 patients and mild 77 patients with normal in 63 patients at visits 2 .At the end of the study 136 patients had become normal, 37 still had mild diarrhoea and 5 patients had moderate. Interestingly none of the patients persisted to have severe diarrhoea at the end of the study.

The mean duration of diarrhea was 5 days in the S. boulardii group. On day 1 stool had a watery, semisolid and soft consistency in 136, 33 and 15 patients respectively. On day 2, 41 patients had watery stool form, 113 patients had a semisolid form ,24 patients had a soft stool form and 5 patients had a Hard stool form. On day 5 there was a reduction in the number of patients having watery stool form coming down to 2 whereas 45 patients had a semisolid stool form ,87 patients had watery stool form and 47 patients had a hard stool form.

Fig 2: Stool consistency on Day1, Day 3 AND Day 5. 


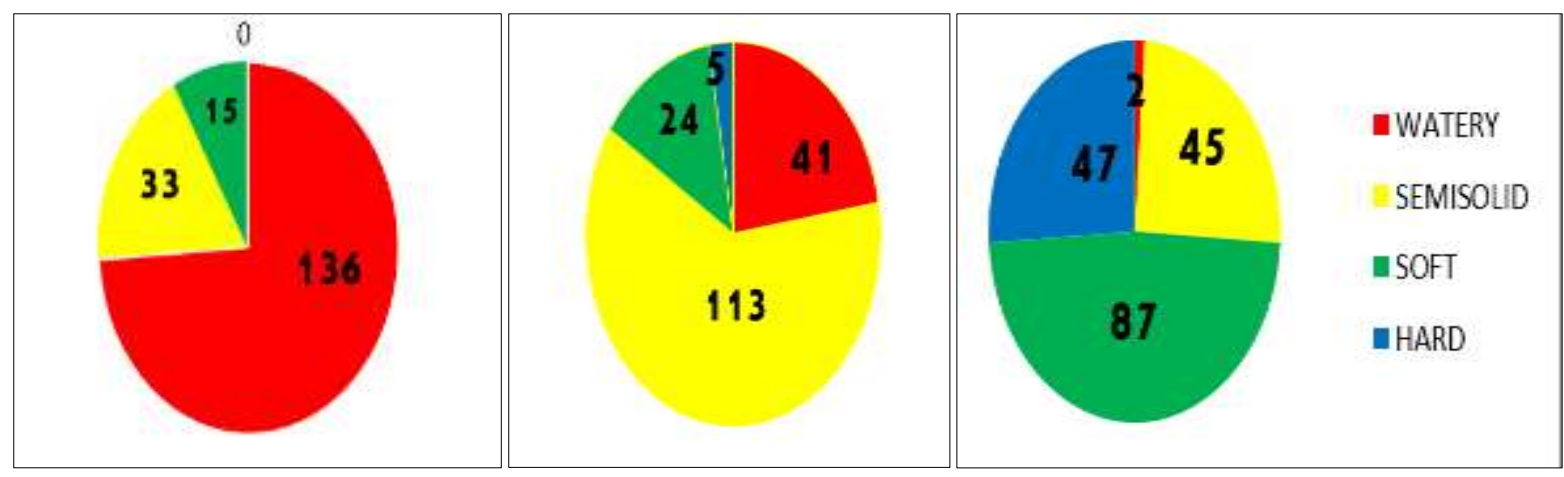

S. boulardii was well accepted and tolerated by the children and there were no reports of any side effects during the study period.

\section{Discussion:}

The intestinal flora is intimately conjoined with the organ which contains it and with which it contour an ecosystem. Equilibrium within this ecosystem is crucial to good health of the individual. It influences the structure, physical, motility and chemical conditions of intestinal tract, enzyme and metabolic activity of mucosa and maturation and establishment of immune system. Above all, the intestinal microbial flora frames a true resistance to colonisation of digestive tract by pathogenic microorganisms. Pathological situations such as infectious diarrhoea or diarrhoea and colitis linked to antibiotic treatment disrupt the intestinal ecosystem.

Probiotics have been used to treat a variety of infections, markedly infections of mucosal surfaces such as the gut. These biotherapeutic agents include the yeast $S$. boulardii. The current study was stationed on our previous clinical observation, which conceded that children treated with $S$. boulardii had a decreased number of episodes of diarrhoea in following months.

The strength of our study is to see the effect of probiotic in the Indian prospect as we may not deduce the results of western population in Indian children due to the higher breast feeding rate and different microbiological colonization due to different food and eating habits.

A Cochrane review analysed 23 studies or meta-analysis of 3938 patients for the treatment of diarrhoea, construing that among the various probiotics evaluated use of Saccharomyces boulardii for preventing AAD in children receiving antibiotics ${ }^{11}$.

AG Billoo et al. ${ }^{13}$ conducted a randomised control clinical trial of S. boulardii in the treatment of diarrhoea. Efficacy and safety of S. boulardii were gauged in 100 children. The study showed a significant improvement in frequency and consistency of stool and thereby verified 50\% reduction in the number of episodes and duration of illness in patients who were given S.boulardii along with WHO.

Villarruel $\mathrm{G}$ et al. ${ }^{14}$, conducted a randomized clinical study with probiotic yeast $\mathrm{S}$. boulardii as an adjuvant to oral rehydration solution (ORS) in shortening the duration of acute infectious gastroenteritis in children less than 2 years old in ambulatory care. The efficacy and safety of S.boulardii were evaluated in 100 patients and were compared with placebo. The risk of having diarrhoea lasting more than 7 days was lower in the $\mathrm{s}$. Boulardii group (3/44 versus $12 / 44$; rr 0.25 ; $95 \%$ ci $0.1-0.8$ ) the study ceased that $\mathrm{s}$. Boulardii as an adjuvant to ors in ambulatory care in children less than 2 years old with mild or moderate acute diarrhoea decreased the duration of diarrhoea, accelerated recovery and reduced the risk of prolonged diarrhoea.

Susrut Das et al. ${ }^{15}$ conducted a double blind randomised control trial to see the Efficacy and Safety of Saccharomyces boulardii in Acute Rotavirus Diarrheal patients. The study showed a significantly shorter Median duration (hours) of diarrhea in the intervention group (60 vs. 89; 95\% CI:_41.2 to_16.8). A significantly shorter duration of hospitalization (74 vs. 91; 95\% CI:_33.46 to_0.54) was also seen in the intervention group. Thus, the study wrapped up to the fact that SB is effective and safe in acute rotavirus diarrhea.

Meeta A. Burande et al. ${ }^{12}$ conducted a parallel, single blind, randomized controlled clinical trial to compare the efficacy of ORS $+\mathrm{Zn}$ verses ORS $+\mathrm{Zn}+$ Probiotics Saccharomyces boulardii strain in treatment of acute diarrhoea in Indian children. The study showed Mean duration of diarrhoea for study group was 3.4 days +1.4 days and for control group was 5.5 days +2.1 days $(Z$ value 4.9$)$. Average time of recovery from vomiting was $2.5+1.2$ days for study group $\&$ for control were $3.3+1.2$ days $(\mathrm{t} 17=3.3, \mathrm{P}<0.01)$. Thus the 
study concluded that addition of Saccharomyces boulardii in the treatment of acute diarrhoea significantly reduce the duration of diarrhoea as well as vomiting.

Several studies of S. boulardii have been done in children and adults in the treatment of acute diarrhoea. The results of our studies are consistent with some of these studies. Stimulation of local immunity, as demonstrated by the increase of IgA, together with the enhancement of the trophic activity of the mucosa (through the release of polyamines) by $S$. boulardii may, at least in part, explain the long term effect of the yeast.

In the current study conducted by the authors, the stool frequency decrease from 9.005 to 2.7 at the end of 5 days. The stool consistency also improved in majority of the patients where the stools had become soft or hard from watery or semisolid.

A meticulous follow-up of the patient resulted in very good compliance. No side effects were observed during the active treatment period with the use of $\mathrm{S}$. boulardii.

\section{Conclusion}

Saccharomyces boulardii is safe and efficacious in the treatment of diarrhoea in Indian children.

\section{Disclosures}

This study was conducted as a part of Pharmacovigilance activity for Floratact Sachet by Centaur Pharmaceuticals Pvt Ltd. in accordance with Pharmacovigilance Program of India (PvPI).

\section{References.}

1. Giannattasio A, Guarino A, Vecchio AL. Management of children with prolonged diarrhea. F1000Research. 2016;5.

2. Lee KJ. Pharmacologic agents for chronic diarrhea. Intestinal research. 2015 Oct 1;13(4):306-12.

3. Lakshminarayanan S, Jayalakshmy R. Diarrheal diseases among children in India: current scenario and future perspectives. Journal of natural science, biology, and medicine. 2015 Jan;6(1):24.

4. REHYDRATION O. Treatment of diarrheal disease.

5. Rao K, Young VB. Fecal microbiota transplantation for the management of Clostridium difficile infection. Infectious disease clinics of North America. 2015 Mar 31;29(1):109-22.

6. Verna EC, Lucak S. Use of probiotics in gastrointestinal disorders: what to recommend?. Therapeutic advances in gastroenterology. 2010 Sep;3(5):307-19.

7. Kechagia M, Basoulis D, Konstantopoulou S, Dimitriadi D, Gyftopoulou K, Skarmoutsou N, Fakiri EM. Health benefits of probiotics: a review. ISRN nutrition. 2013 Jan 2;2013.

8. Guandalini S. Probiotics for prevention and treatment of diarrhea. Journal of Clinical Gastroenterology. 2011 Nov 1;45:S149-53.

9. Stier H, Bischoff SC. Influence of Saccharomyces boulardii CNCM I-745on the gut-associated immune system. Clinical and experimental gastroenterology. 2016;9:269.

10. https://www.probiotaevent.com/prof-gregor-reid/

11. Johnston BC, Supina AL, Vohra S. Probiotics for pediatric antibiotic-associated diarrhea: a metaanalysis of randomized placebo-controlled trials. Canadian Medical Association Journal. 2006 Aug $15 ; 175(4): 377-83$.

12. Dinleyici EC, Eren M, Ozen M, Yargic ZA, Vandenplas Y. Effectiveness and safety of Saccharomyces boulardii for acute infectious diarrhea. Expert opinion on biological therapy. 2012 Apr 1;12(4):395-410.

13. Billoo AG, Memon MA, Khaskheli SA, Murtaza G, Iqbal K, Shekhani MS, Siddiqi AQ. Role of a probiotic (Saccharomyces boulardii) in management and prevention of diarrhoea. World journal of gastroenterology: WJG. 2006 Jul 28;12(28):4557.

14. Villarruel G, Rubio DM, Lopez F, Cintioni J, Gurevech R, Romero G, Vandenplas Y. Saccharomyces boulardii in acute childhood diarrhoea: a randomized, placebo-controlled study. Acta Paediatrica. 2007 Apr 1;96(4):538-41.

15. Das S, Gupta PK, Das RR. Efficacy and safety of saccharomyces boulardii in acute rotavirus diarrhea: double blind randomized controlled trial from a developing country. Journal of tropical pediatrics. 2016 Jun 9;62(6):464-70. 